\title{
Antioxidants inhibit subsequent lipid production via sebaceous gland cell differentiation
}

\begin{abstract}
Objective: The effects of sebum lipid production by sebaceous gland cells were investigated in vitro following inhibition of cell differentiation by the antioxidants (ascorbyl/tocopheryl) phosphoric acid, ascorbic acid, and pyridoxine cyclic phosphate.

Methods: Normal hamster sebaceous gland cells were exposed to various concentrations of the antioxidants for 10 days. Lipids were extracted from the cells and quantitated by high-performance thin-layer chromatography. Numbers of lipidcontaining differentiated cells were determined by light microscopy. The superoxide anion scavenging ability of each antioxidant was measured by the nitro blue tetrazolium method.

Results: All antioxidants suppressed sebocyte cell differentiation and decreased levels of sebum lipid secretion, with (ascorbyl/tocopheryl) phosphoric acid being the most effective. Antioxidant ability, the number of differentiated sebocyte cells, and sebum secretion level were found to highly correlate.
\end{abstract}

Conclusion: These findings indicate that antioxidant compounds inhibit sebaceous lipid production by suppressing differentiation of sebocytes.

Keywords: sebocyte, lipid, antioxidant, differentiated cell, high-performance thinlayer chromatography

\author{
Volume I Issue 3 - 2017 \\ Yoshihiro Tokudome, Yoshimi Takahashi \\ Department of Pharmaceutical Technochemistry, Josai \\ University, Japan
}

Correspondence:Yoshihiro Tokudome, Department of Pharmaceutical Technochemistry, Josai University, Japan, Tel +8I49-27I-8I40, Email tokudome@josai.ac.jp

Received: August 12, 2017 | Published: September 05, 2017
Abbreviations: EDTA, ethylene diaminete traacetic acid; NBT, nitrotetrazolium blue tetrazolium; PBS, phosphate buffered saline; XOD, xanthine oxidase

\section{Introduction}

The sebaceous gland is composed of sebaceous gland cells that accumulate intracellular lipid droplets when undergoing differentiation. The extent of lipid droplet formation within the sebaceous gland cells thus represents their differentiation status. On reaching maturity, the sebaceous gland cells are then destroyed and the intracellular lipids are secreted to the skin surface whereby they form a weakly acidic sebum film with intercellular lipids of the stratum corneum and sweat. The main sebum lipid is composed of triglycerides, including cholesterol, free fatty acids and other members. Sebum prevents invasion of foreign matter from the outside and protects the skin from irritation of various substances. It also has a moisture retention function and sterilization action. In atopic dermatitis patients, the amount of sebum decreases, which is thought to be caused by decreased sebum production and/or secretion. Excessive secretion of sebum is associated with skin diseases such as acne and lipid peroxide production. ${ }^{1}$ In addition, secretion of sebum is promoted not only by environmental factors such as ultraviolet (UV) rays (predominantly UVB) and temperature rise, but also by external factors such as gamma oryzanol, herbal extracts (liquorice, carrot, yokuinin), and endogenous factors such as androgen hormones. ${ }^{2,3}$ Furthermore, lipid peroxide, which is also increased by UV rays (UVB) and secreted to the skin surface, irradiated UV rays (UVB), and lipid peroxides destroy the skin barrier function. Testosterone, an androgenic hormone, is converted to dihydrotestosterone by 5-reductase, which stimulates the sebaceous glands and increases sebum secretion. Furthermore, these components increase the sebaceous gland size, ${ }^{4-6}$ cell division ${ }^{7}$ and sebum production. ${ }^{8,9}$ Interestingly, secretion of sebum is promoted by UV rays (UVB) and male hormones ${ }^{10,11}$ butis suppressed by pyridoxine or retinoic acid.

Antioxidants suppress generation of reactive oxygen species ${ }^{12}$ and therefore control oxidative stress, which is a key factor for the onset and exacerbation of many diseases. Antioxidant substances in the bodyinclude oxidative enzymes (superoxide dismutase, catarase), vitamins (ascorbic acid, tocopherol), carotenoids (astaxanthin, B-carotene), coenzyme Q10, and fullerenes, to name a few. Of note, sebum production inhibition by fullerene has been reported in vitro; however, its usefulness has not been fully discussed. ${ }^{13}$

In this study, we investigated the potential antioxidant effects of (ascorbyl/tocopheryl) phosphoric acid, ascorbic acid, and pyridoxine cyclic phosphate on inhibition of sebaceous gland cell differentiation and sebum production.

\section{Materials and methods}

\section{Cells and culture medium}

Normal hamster sebaceous gland cells (KB-4009), sebocyte cell proliferation medium (Humedia-BG medium), and sebaceous gland cell differentiation medium (Humedia-BD medium) were purchased from Kurabo Co., Ltd. (Osaka, Japan).

\section{Test compounds}

(Ascorbyl/tocopheryl) phosphoric acid was purchased from Senju Pharmaceutical Co., Ltd. (Osaka, Japan). Ascorbic acid was purchased from Wako Pure Chemical Industries, Ltd. (Osaka, Japan). Pyridoxine 
cyclic phosphate was purchased from Daiichi Fine Chemical Co., Ltd. (Takaoka, Toyama, Japan).

\section{Reagents}

Chloroform, methanol, cholesterol, hexane, diethyl ether, acetic acid, copper sulfate, and phosphoric acid were purchased from Wako Pure Chemical Industries, Ltd. Triolein, palmitic acid, nitrotetrazolium blue tetrazolium (NBT), hypoxanthine, ethylenediaminetetraacetic acid (EDTA), and xanthine oxidase (XOD) were purchased from Sigma Aldrich (St. Louis, MO, USA).

\section{Cell culture}

Normal hamster sebaceous gland cells were seeded at $1.0 \times 10^{4} \mathrm{cells} /$ well in a 24-well plate and incubated at $37^{\circ} \mathrm{C}$ in $5 \% \mathrm{CO}_{2}$ using Humedia-BG medium.

\section{Sample addition}

Arbitrary concentrations of (ascorbyl/tocopheryl) phosphoric acid, ascorbic acid and pyridoxine cyclic phosphate were prepared in Humedia-BD medium and added to the culture medium every 2 days for 10 days. Figure 1 shows the structure of the three compounds used in this study.

(a)

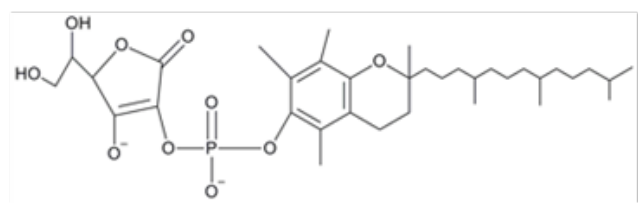

(b)<smiles>O=C1OC(C(O)CO)C(O)=C1O</smiles>

(c)

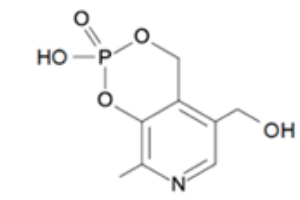

Figure I Antioxidant chemical structures. (Ascorbyl/tocopheryl) phosphoric acid (A), ascorbic acid (B), and pyridoxine cyclic phosphate (C).

\section{Extraction of intracellular lipid content}

Lipid extraction was carried out by modifying the Bligh and Dyer method. ${ }^{14}$ After cell culture, the medium was removed and the cells were washed with PBS. Purified water $(0.5 \mathrm{~mL})$ was then added and the cells were collected in a test tube using a cell scraper. Chloroform $(1.5 \mathrm{~mL})$ and methanol $(3.0 \mathrm{~mL})$ were then added and stirred. Cells were disrupted under output control 2 and duty cycle 50 for 10 seconds using an ultrasonic homogenizer (Advanced Sniffer 250, Branson Ultrasonics, Danbury, CT, USA). Filtration was carried out with a filter (Millex GN $0.20 \mu \mathrm{m}$, Millipore, Billerica, MA, USA) attached to a glass syringe to remove cell debris. Purified water $(1.5 \mathrm{~mL})$ was then added so that the final ratio of chloroform: methanol: purified water was 2:2:1, and the mixture was stirred. Centrifugation (HIMAC 11
CT GE, Hitachi, Tokyo, Japan) was then performed at 1100rpm for 20minutes at room temperature to separate the chloroform and water into two layers. The upper layer was removed and the lower layer was dried under a nitrogen stream using a dry block bath (Dry Thermo Unit DTU-1C, Taitec, Koshigaya, Saitama, Japan). Thereafter, it was re-dissolved in $100 \mu \mathrm{L}$ of chloroform: methanol $(2: 1)$ and used as a sample.

\section{Lipid analysis}

The sample $(10 \mu \mathrm{L})$ was spotted on a high-performance thin-layer chromatography plate (Silica Gel 60, Merck, Darmstadt, Germany) using a glass capillary (ringcaps, Hirschman Laboratorate, Eberstadt, Postfach, Germany).This was then developed with hexane: diethyl ether: acetic acid (80:20:1) for 15 minutes, dried and re-developed in an identical manner. Thereafter, the solution was sprayed with an aqueous solution of $8 \%$ phosphoric acid containing $10 \%$ copper sulfate and heated at $200^{\circ} \mathrm{C}$ for 5 minutes using a TLC plate heater III (CAMAG, Sonnenmattstrasse, Muttenz, Switzerland). This was imaged with a Lumino image analyzer (LAS-1000 plus, Fuji Film Co., Tokyo, Japan) and the amount of each lipid was quantified from density using a Multi Gauge (Fuji Film Co., Tokyo, Japan). Quantification was carried out from a calibration curve of each lipid concentration and density.

\section{Observation of cell morphology and measurement of differentiated cell number}

After cell culture, the medium was removed and replaced with PBS and cell morphology was observed using an inverted microscope (IX 71, Olympus, Tokyo, Japan) and imaged using DP2-BSW software (Olympus). The number of differentiated cells contained in $15.3 \times 20.5$ pixels was counted and corrected by area to calculate the number of differentiated cells per well.

\section{Count of differentiated cell number}

After adding each concentration of (ascorbyl/tocopheryl) phosphate, ascorbic acid, and pyridoxine cyclic phosphate to normal hamster sebaceous gland cells every two days for 10 days, cell morphology was imaged using an inverted microscope and the number of differentiated cells was counted.

\section{Measurement of the superoxide anion scavenging ability}

Reaction buffer $(75 \mu \mathrm{L} /$ well) containing $0.25 \mathrm{mM}$ NBT, $1.0 \mathrm{mM}$ hypoxanthine, and $0.1 \mathrm{mM}$ EDTA was placed into wells a 96well microplate. Then $0.01,0.1,1.0$, and $5.0 \mathrm{mM}$ of either ascorbic acid, (ascorbyl/tocopheryl) phosphoric acid, or pyridoxine cyclic phosphate, each with the addition of 20units/mL XOD solution, were added to wells and mixed. The plate was then transferred to a microplate reader (Spectra Max M2 ${ }^{\mathrm{e}}$, Molecular Devices, Silicon Valley, CA, USA), and the absorbance of formazan was measured at $550 \mathrm{~nm}$. In order to exclude influence of the sample on the absorbance at the time of measurement, a sample of each concentration was prepared by replacing the XOD solution with PBS (reaction buffer $75 \mu \mathrm{L}$, sample $25 \mu \mathrm{L}$, PBS $25 \mu \mathrm{L}$ ) and absorbance was measured in the same manner. The superoxide anion scavenging ability was calculated using the following equation:

Superoxide anion scavenging ability $(\%)=1-\frac{A b s(\text { sample })-A b s(X O D=0)}{A B S(\text { sample }=0)-A b s(X O D=0)} \times 100$ 


\section{Data analysis}

The data are expressed as means \pm standard deviations (SD) of three experiments and compared to the control group by Dunnett's test using SAS University Edition software (SAS Institute Inc., Cary, NC, USA).

\section{Results}

\section{Effect on intracellular lipid content}

Figure 2 shows the effect on intracellular lipid content. (Ascorbyl/ tocopheryl) phosphoric acid significantly decreased intracellular lipid content in a dose-dependent manner $(p<0.01)$. Addition of ascorbic acid and pyridoxine cyclic phosphate did not significantly change the intracellular lipid level.
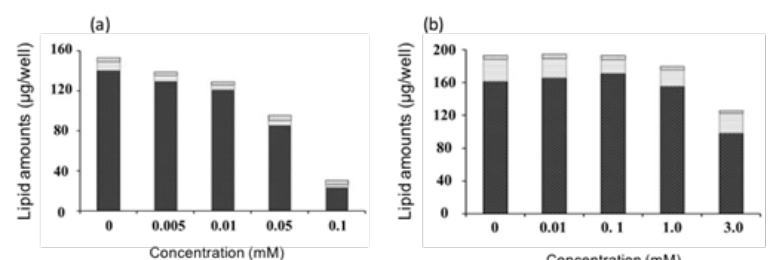

(c)

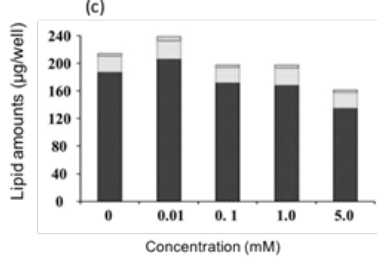

Figure 2 Effect of antioxidants on lipid accumulation in cultured hamster sebocytes. Triglyceride (black bar), free fatty acid (white bar), cholesterol (vertical line bar). (Ascorbyl/tocopheryl) phosphate (A), ascorbic acid (B), and pyridoxine cyclic phosphate $(C)$. Values represent means $\pm S . D$. of three experiments, ${ }^{*} \mathrm{p}<0.05,{ }^{* *} \mathrm{p}<0.0$ I versus control $(0 \mathrm{mM})$; Dunnett's test.

\section{Effect of antioxidants on differentiated cell number} and cell morphology

Figure 3 show the differentiated cell number and cell morphology after exposure to each antioxidant. (Ascorbyl/tocopheryl) phosphate decreased the number of differentiated cells in a dose-dependent manner. Ascorbic acid and pyridoxine cyclic phosphate showed a decreasing trend in the number of differentiated cells dependent on concentration. Exposure to (ascorbyl/tocopheryl) phosphate resulted in the highest number of proliferating cells and greatest suppression of differentiation. All the antioxidants decreased the number of differentiated cells with increased concentration (Figure 4). In particular (ascorbyl/tocopheryl) phosphate at the lowest concentration resulted in the greatest reduction in the number of differentiated cells.

\section{Measurement of superoxide anion scavenging ability}

All antioxidants tested resulted in an enhanced superoxide anion elimination rate in a concentration-dependent manner (Figure 5). In particular, (ascorbyl/tocopheryl) phosphate resulted in the highest superoxide anion elimination rate. The relationship among the various antioxidant substances with respect to sebum production suppression, cell differentiation inhibition, and antioxidant ability (superoxide anion scavenging ability) are apparent from these findings, whereby intracellular lipid content was decreased with increased antioxidant capacity (superoxide anion scavenging ability)and in proportion to the number of differentiated cells (Figure 6). Furthermore, the number of differentiated cells decreased with increased antioxidant capacity (superoxide anion scavenging ability).
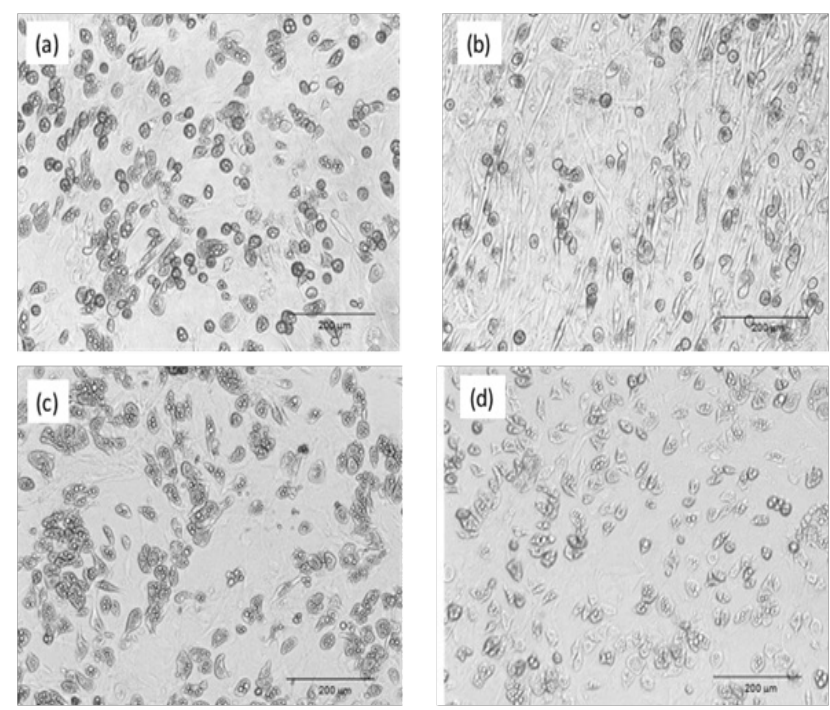

Figure 3 Effect of antioxidants on sebaceous gland cell differentiation. Control (A); (ascorbyl/tocopheryl) phosphate (B); ascorbic acid (C); and pyridoxine cyclic phosphate (D).
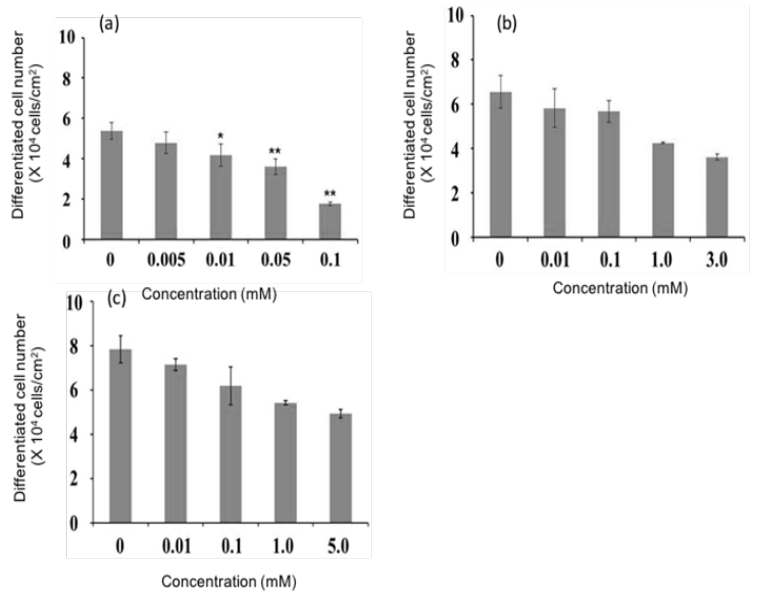

Figure 4 Effect of antioxidants on differentiated cell number. (Ascorbyl/ tocopheryl) phosphate (A); ascorbic acid (B); and pyridoxine cyclic phosphate (C). Values represent means \pm S.D. of three experiments, ${ }^{*} p<0.05,{ }^{*} p<0.01$, versus control (0mM each sample); Dunnett's test.

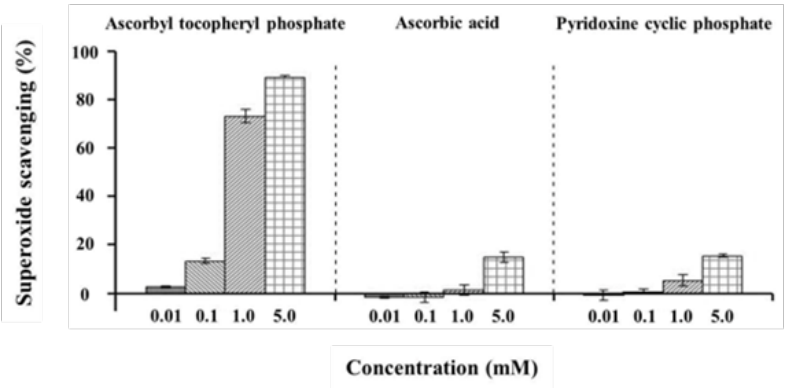

Figure 5 Effect of antioxidants on the superoxide scavenging ability. Values represent means \pm S.D. of three experiments, ${ }^{*} \mathrm{p}<0.05$, ${ }^{*} \mathrm{p}<00.0 \mathrm{I}$, versus control (OmM); Dunnett's test. 
(a)

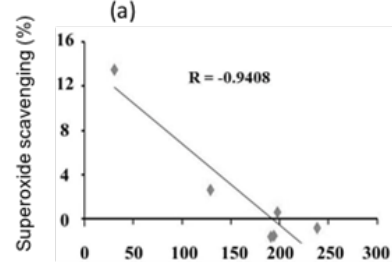

Lipid amount ( $\mu \mathrm{g} / \mathrm{well})$

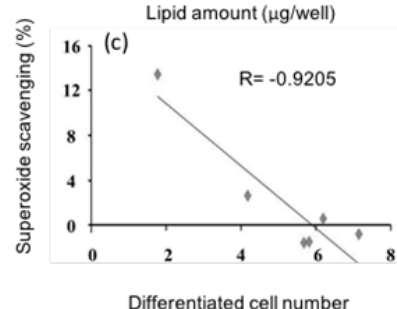

(X $10^{4}$ cells $\left./ \mathrm{cm}^{2}\right)$

Figure 6 Relationships between lipid amount, differentiated cell number, and superoxide scavenging ability. Relationship between lipid amount and superoxide scavenging ability (A). Relationship between lipid amount and differentiated cell number (B). Relationship between differentiated cell number and superoxide scavenging ability $(C)$.Values represent the average of three experiments.

\section{Discussion}

Following sebaceous gland cell proliferation, subsequent cell differentiation results in the formation of intracellular sebumcontaining lipid droplets. ${ }^{15,16}$ On maturation, the cell ruptures and the sebum is secreted to the skin surface. ${ }^{17,18}$ Because the amount of sebum varies depending on the proliferation and differentiation status of the sebaceous gland cells, we hypothesized that suppression of sebaceous gland cell differentiation would reduce the amount of sebum. In this study, we investigated the influence of various antioxidants on sebaceous gland cells with respect to intracellular lipid content, cell differentiation, and antioxidant power.

First, the effects of various antioxidants on the intracellular lipid content of sebaceous gland cells were examined.(Ascorbyl/tocopheryl) phosphate $(0-0.1 \mathrm{mM})$, ascorbic acid $(0-3.0 \mathrm{mM})$, and pyridoxine cyclic phosphate $(0-5 \mathrm{mM})$ were added to normal hamster sebaceous gland cells every 2 days for 10 days, and the intracellular lipid content was quantified. Antioxidant concentrations were not affected by the cells. As a result, (ascorbyl/tocopheryl) phosphoric acid significantly decreased the intracellular lipid content with increased concentration, suggesting that antioxidants suppress sebum production. Interestingly, antioxidant substances are reported to promote the proliferation of normal human keratinocytes and in some cases promote/suppress their differentiation. ${ }^{19,20}$

Next, the influence of various antioxidants on sebaceous gland cell morphology and differentiated cell number was examined. Addition of (ascorbyl/tocopheryl) phosphate resulted in a greater number of cells undergoing proliferation with reduced differentiation. All the antioxidants reduced the number of differentiated cells with increased concentration. These findings indicate that the antioxidants suppress sebaceous lipid production by inhibiting cell differentiation. Interestingly, ascorbic acid is reported to promote epidermal keratinocyte cell differentiation without promoting proliferation in vitro. ${ }^{21}$ Therefore, antioxidant substances are thought to suppress sebum production without adversely affecting turnover of the epidermis, which is important for maintaining skin homeostasis. Finally, as a result of evaluating the antioxidant power of antioxidants, superoxide anion scavenging ability was increased in a concentrationdependent manner, with (ascorbyl/tocopheryl) phosphate resulting the highest superoxide anion scavenging ability.

The effects of inhibiting sebum production, suppressing cell differentiation, and antioxidant capacity (superoxide anion eliminating ability) of these antioxidants are therefore more clearly defined from this study. Intracellular lipid content decreased with increased antioxidant capacity (superoxide anion scavenging ability) and decreased in proportion to the number of differentiated cells. Furthermore, the number of differentiated cells decreased with increased antioxidant capacity (superoxide anion eliminating ability). This suggests correlation exists between antioxidant power of the compound, cell differentiation status, and sebum production.

From these findings, it is assumed that the antioxidant capacity (superoxide anion eliminating ability) of the compound is related to cell differentiation and sebum amount, and therefore stronger antioxidants like (ascorbyl/tocopheryl) phosphate inhibit cell differentiation to a greater extent and thus the production of sebum. However, because the exact mechanism of sebaceous gland cell differentiation inhibition remains unknown, it is necessary to clarify this in future studies. It is also unclear whether the inhibition cell differentiation and sebum production by (ascorbyl/tocopheryl) phosphate acts through (ascorbyl/ tocopheryl) phosphate or acts through ascorbic acid and tocopherol. Therefore, further study on this is necessary. Finally, because the method used in this study cannot measure the antioxidant ability of hydrophobic substances, it is necessary to develop a method of measuring this and determine the effect of these on cells.

\section{Conclusion}

These findings indicate that antioxidant compounds inhibit sebaceous lipid production by suppressing differentiation of sebocyte.

\section{Acknowledgements}

None.

\section{Conflict of interest}

The author declares no conflict of interest.

\section{References}

1. Akitomo Y, Akamatsu H, Okano Y, et al. Effects of UV irradiation on the sebaceous gland and sebum secretion in hamsters. J Dermatol Sci. 2003;31(2):151-159.

2. Lesnik RH, Kligman LH, Kligman AM. Agents that cause enlargement of sebaceous glands in hairless mice. I. Topical substances. Arch Dermatol Res. 1992;284(2):100-105.

3. Lesnik RH, Kligman LH, Kligman AM. Agents that cause enlargement of sebaceous glands in hairless mice. II. Ultraviolet radiation. Arch Dermatol Res. 1992;284:106-108.

4. Ebling FJ, Ebling E, McCaffery V, et al. The response of the sebaceous glands of the hypophysectomized-castrated male rat to 5 -dihydrotestosterone, androstenedione, dehydroepiandrosterone and androsterone. J Endocrinol. 1971;51:181-190. 
5. Ebling FJ, Ebling E, McCaffery V, et al. The responses of the sebaceous glands of the hypophysectomized-castrated male rat to $5 \alpha$-androstanedione and 5 $\alpha$-androstane-3 $\beta, 17 \beta$-diol. J Invest Dermatol. 1973;60(4):183-187.

6. Thody AJ, Cooper MF, Bowden PE, et al. Effect of alpha-melanocytestimulating hormone and testosterone on cutaneous and modified sebaceous glands in the rat. J Endocrinol. 1976;71(3):279-288.

7. Sauter LS, Loud AV. Morphometric evaluation of sebaceous gland volume in intact, castrated, and testosterone-treated rats. J Invest Dermatol. 1975;64(1):9-13.

8. Burgess TL, Wilson JD. Studies on hormonal regulation of squalene synthesis in preputial gland and skin of the rat. Proc Soc Exp Biol Med. 1963;113:747-750.

9. Sansone G, Davidson W, Cummings B, et al. Sebaceous gland lipogenesis induced by testosterone: early metabolic events. J Invest Dermatol. 1971;57(3):144-148.

10. Zouboulis CC, Xia L, Akamatsu H, et al. The human sebocyte culture model provides new insights into development and management of seborrhoea and acne. Dermatology (Basel). 1998;196(1):21-31.

11. Ito A, Sakiguchi T, Kitamura K, et al. Establishment of a tissue culture system for hamster sebaceous gland cells. Dermatology (Basel). 1998;197:238-244.

12. Masaki H. Role of antioxidants in the skin: anti-aging effects. J Dermatol Sci. 2010;58(2):85-90.

13. Inui S, Aoshima H, Nishiyama A, et al. Improvement of acne vulgaris by topical fullerene application: unique impact on skin care. Nanomedicine. 2011;7(2):238-241
14. Bligh EG, Dyer WJ. A rapid method of total lipid extraction and purification. Can J BiochemPhysiol. 1959;37(8):911-917.

15. Wheatley VR, Potter JER, Lew G. Sebaceous gland differentiation: II. The isolation, separation and characterization of cells from the mouse preputial gland. J Invest Dermatol. 1979;73(4):291-296.

16. Akimoto N, Sato T, Sakiguchi $\mathrm{T}$, et al. Cell proliferation and lipid formation in hamster sebaceous gland cells. Dermatology (Basel). 2002;204(2):118-123

17. Shelmire JB Jr. Some Interrelations between sebum, sweat and the skin surface. J Invest Dermatol. 1959;32(3):471-472.

18. Dvorken L, Maggiora A, Jadassohn W. The Problem of sebum spread on the surface of the skin. Dermatology (Basel). 1966;132:59-63.

19. Balasubramanian S, Eckert R. Keratinocyte proliferation, differentiation, and apoptosis-Differential mechanisms of regulation by curcumin, EGCG and apigenin. Toxicol Appl Pharmacol. 2007;224(3):214-219.

20. An J, Li Z, Dong Y, et al. Amentoflavone protects against psoriasis-like skin lesion through suppression of NF- $\kappa \mathrm{B}$-mediated inflammation and keratinocyte proliferation. Mol Cell Biochem. 2016;413(1-2):87-95.

21. Savini I, Catani MV, Rossi A, et al. Characterization of keratinocyte differentiation induced by ascorbic acid: protein kinase $\mathrm{C}$ involvement and vitamin C homeostasis. J Invest Dermatol. 2002;118(2):372-379. 